\title{
CHARACTERIZATION OF A PROTOTYPE RADIO FREQUENCY SPACE ENVIRONMENT PATH EMULATOR FOR EVALUATING SPACECRAFT RANGING HARDWARE*
}

\author{
Jason W. Mitchell ${ }^{\dagger}$ and Philip J. Baldwin ${ }^{\ddagger}$ \\ Emergent Space Technologies, Inc., Greenbelt, MD 20770-6334, USA \\ Rishi Kurichh§ ${ }^{\S}$ Bo J. Naasz $\$$, and Richard J. Luquettell \\ NASA Goddard Space Flight Center, Greenbelt, MD 20771, USA
}

\begin{abstract}
The Formation Flying Testbed (FFTB) at the National Aeronautics and Space Administration (NASA) Goddard Space Flight Center (GSFC) provides a hardware-in-theloop test environment for formation navigation and control. The facility is evolving as a modular, hybrid, dynamic simulation facility for end-to-end guidance, navigation and control (GN\&C) design and analysis of formation flying spacecraft. The core capabilities of the FFTB, as a platform for testing critical hardware and software algorithms in-the-loop, have expanded to include S-band Radio Frequency (RF) modems for interspacecraft communication and ranging. To enable realistic simulations that require $\mathrm{RF}$ ranging sensors for relative navigation, a mechanism is needed to buffer the RF signals exchanged between spacecraft that accurately emulates the dynamic environment through which the RF signals travel, including the effects of the medium, moving platforms, and radiated power. The Path Emulator for Radio Frequency Signals (PERFS), currently under development at NASA GSFC, provides this capability. The function and performance of a prototype device are presented.
\end{abstract}

Keywords: radio frequency signals, spacecraft crosslinks, relative navigation, delay, signal buffering, real-time, hardware-in-the-loop, formation flying, formation control.

$\begin{array}{ll}\text { ADC } & \text { Analog-to-Digital Converter } \\ \text { CA } & \text { Carrier Acquisition } \\ \text { CCS } & \text { Crosslink Channel Simulator } \\ \text { DAC } & \text { Digital-to-Analog Converter } \\ \text { DDS } & \text { Direct Digital Synthesizer }\end{array}$

*This material is declared a work of the U.S. Government and is not subject to copyright protection in the United States.

${ }^{\dagger}$ Aerospace Scientist, jason.mitchell@emergentspace.com, AIAA Senior Member.

$¥$ Assoc. Software Engineer, philip. baldwin@emergentspace.com.

${ }^{\S}$ Computer Engineer, Code 596, rishi.kurichh-1@nasa.gov.

"Aerospace Engineer, Code 595, bo.naasz@nasa.gov, AIAA Member.

" Aerospace Engineer, Project PI, Code 591, rich.luquette@nasa.gov, AIAA Member. 


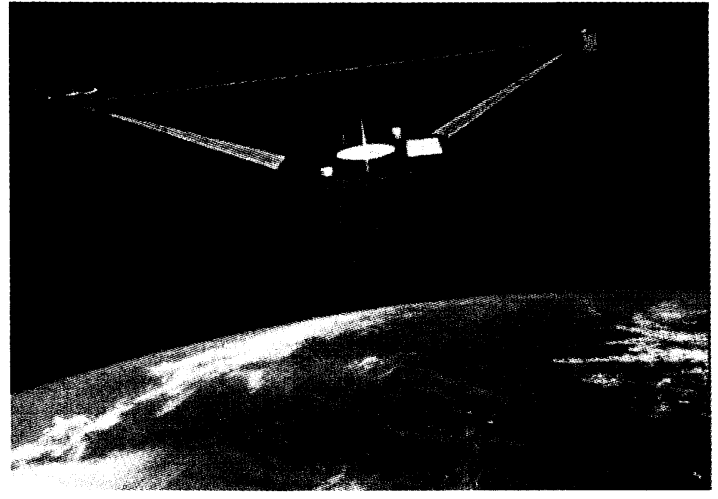

Figure 1. Artist's concept of formation flying spacecraft exchanging information via crosslink.

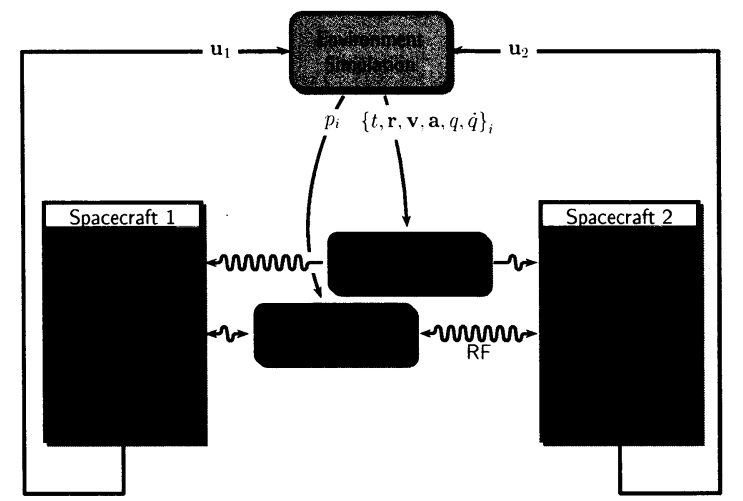

Figure 2. Example connectivity for a two satellite Earth orbiting simulation employing GPS and RF crosslink ranging.

$\begin{array}{ll}\text { DSA } & \text { Digital Step Attenuator } \\ R_{\oplus} & \text { Earth Radii } \\ \text { ESA } & \text { European Space Agency } \\ \text { FFTB } & \text { Formation Flying Testbed } \\ \text { FPGA } & \text { Field Programmable Gate Array } \\ \text { GEONS } & \text { GPS Enhanced On-Board Navigation System } \\ \text { GN\&C } & \text { Guidance, Navigation, \& Control } \\ \text { GPS } & \text { Global Positioning System } \\ \text { GSFC } & \text { Goddard Space Flight Center } \\ \text { IF } & \text { Intermediate Frequency } \\ \text { IRAS } & \text { Inter-spacecraft Ranging and Alarm System } \\ \text { MMS } & \text { Magnetospheric Multiscale } \\ \text { NASA } & \text { National Aeronautics and Space Administration } \\ \text { PERFS } & \text { Path Emulator for Radio Frequency Signals } \\ \text { PRN } & \text { Pseudo-Random Noise } \\ \text { RF } & \text { Radio Frequency } \\ \text { SI } & \text { Stellar Imager } \\ \text { SPECS } & \text { Submillimeter Probe of the Evolution of Cosmic Structure } \\ \text { ST9 } & \text { Space Technology 9 } \\ \text { TRL } & \text { Technology Readiness Level } \\ \text { VCP } & \text { Virtual COM Port }\end{array}$

\section{Introduction}

CPACECRAFT formation flying is a concept that continues to attract significant attention; PFigure 1. The President's Commission on Implementation of United States Space Exploration Policy ${ }^{1}$ identifies formation flying as one of seventeen enabling technologies needed to meet exploration objectives.

Both the National Aeronautics and Space Administration (NASA) and the European Space Agency (ESA) are evaluating formation flying concepts for numerous planned missions. A brief list of currently planned missions include, NASA: Magnetospheric Multiscale 
(MMS), ${ }^{2}$ Black Hole Imager ${ }^{3,4}$ Submillimeter Probe of the Evolution of Cosmic Structure (SPECS) ${ }^{5}$ Stellar Imager (SI) ${ }^{6}$ ESA: Darwin,${ }^{7}$ Prisma,${ }^{8}$ Proba- $3 .{ }^{9}$ In addition, precision formation flying was evaluated as one of the five candidate technology capability areas for the New Millennium Program's Space Technology 9 (ST9) Project. ${ }^{10,11}$

The Formation Flying Testbed (FFTB $)^{12-14}$ at NASA Goddard Space Flight Center (GSFC) provides a unique environment for designing and testing formation flying Guidance, Navigation, \& Control (GN\&C) algorithms. The FFTB enables an end-to-end capability by testing GN\&C algorithms in real-time, and in the presence of essential flight hardware, e.g. relative navigation sensors and crosslink transceivers. By including this hardware directly in the closed-loop testing, researchers and engineers gain valuable information about the interaction and performance of their algorithms and essential hardware.

In support of the MMS mission, NASA GSFC is maturing the Inter-spacecraft Ranging and Alarm System (IRAS) to Technology Readiness Level (TRL) ${ }^{15,16} 6^{* *}$. The MMS mission is composed of a four satellite formation that is currently scheduled for launch in 2014. The formation will progress through a variety of highly eccentric orbits with perigee altitude of approximately 1.2 Earth Radii $\left(R_{\oplus}\right)$, and apogee altitude between $12 R_{\oplus}$ to $25 R_{\oplus}$ depending on mission phase. ${ }^{17}$ The IRAS serves a number of critical roles for this mission, including: Global Positioning System (GPS) and crosslink ranging; on-board absolute and relative orbit determination using the GPS Enhanced On-Board Navigation System (GEONS); ${ }^{18}$ crosslink communications for navigation information sharing; and science alarm messaging.

The core capabilities of the FFTB, as a platform for testing critical hardware and software algorithms in-the-loop, have expanded to include S-band Radio Frequency (RF) modems for inter-spacecraft communication and ranging. To enable realistic simulations that require $\mathrm{RF}$ ranging sensors for relative navigation, a mechanism is needed to buffer the RF signals exchanged between spacecraft that accurately emulates the dynamic environment through which those signals travel, including the effects of the medium, moving platforms, and radiated power.

In previous work, Hunt et al. ${ }^{19}$ describe a Crosslink Channel Simulator (CCS). This device was successfully integrated into the FFTB and provides a single, dynamic, bi-directional RF path between two spacecraft. Mitchell and Luquette ${ }^{14}$ and Mitchell et al. ${ }^{20}$ describe two spacecraft ST9 scenarios, Figure 2, in which the CCS was the medium for communications via hardware crosslinks. In these scenarios, the hardware crosslinks were used to exchange data between spacecraft, e.g. pseudorange and simulated range measurements, however no direct RF ranging was performed using hardware crosslinks.

Since the MMS mission is composed of four spacecraft, each with multiple antennæ, the IRAS TRL 6 testing in the FFTB will require multiple bi-directional channels. The Path Emulator for Radio Frequency Signals (PERFS), currently under development at NASA GSFC, will provide this additional RF channel capacity. Each PERFS device will provide a single, dynamic, bi-directional, RF path between two spacecraft, much like the CCS. However, the PERFS will have a smaller footprint and lower cost than the CCS, and provide additional testing flexibility. In the following, we present the design concept and performance analysis of a prototype PERFS device.

\footnotetext{
**TRL 6 represents a successful system or subsystem model or prototype demonstration in a relevant environment.
} 


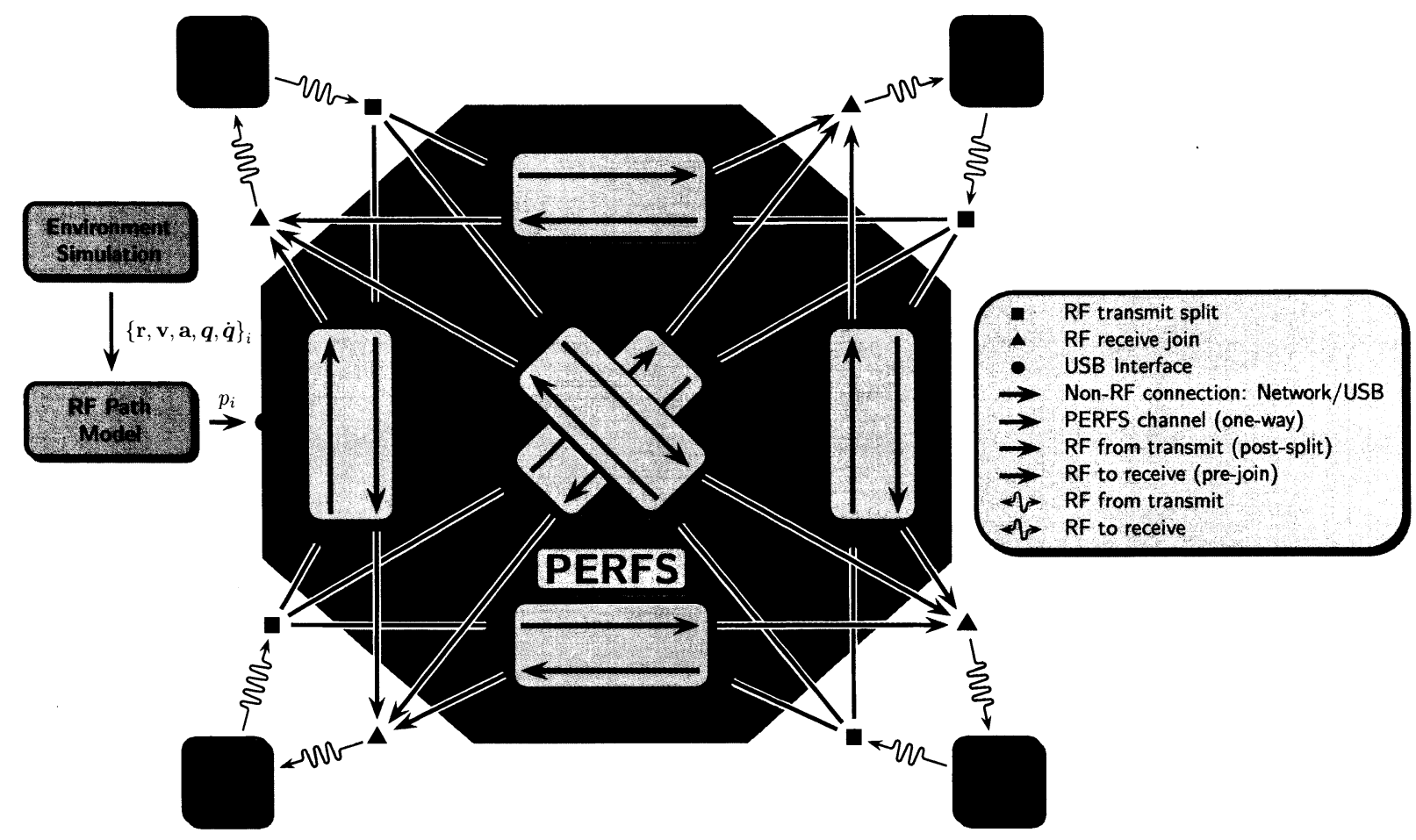

Figure 3. PERFS connectivity for IRAS testing.

\section{Design Concept}

The planned MMS mission consists of four (4) identical spacecraft equipped with an IRAS. To maintain the relative tetrahedral formation, an individual spacecraft must communicate with its three (3) neighbors. Thus, for ground testing and simulation of the complete system, six (6) total bi-directional paths are necessary to enable communication between any single spacecraft and the remaining three (3) spacecraft.

For IRAS TRL 6 testing, the RF environment emulation is embedded into the FFTB simulation architecture as seen in Figure 3. Each IRAS device under test is inter-connected through PERFS as required for RF communication. Spacecraft information, including position, velocity, attitude, acceleration, attitude rate, and antenna pattern, flows into the RF path model. This model computes the parameters, $p_{i}$, which specify the RF environment on each PERFS channel resulting from effects of the medium, moving platforms, and radiated power. Table 1 provides general performance indicators for PERFS.

The PERFS design concept for dynamically buffering RF signals is straightforward, see Figure 4. Flowing from top-left to bottom-right, the S-band RF center frequency input,

\begin{tabular}{cccc}
\hline \hline & Min & Max & Resolution \\
\hline Attenuation & $90 \mathrm{~dB}$ & $0 \mathrm{~dB}$ & $0.5 \mathrm{~dB}$ \\
Range & $100 \mathrm{~m}$ & $3500 \mathrm{~km}$ & $5 \mathrm{~cm}$ \\
Doppler & $0 \mathrm{~Hz}$ & $5 \mathrm{kHz}$ & $10 \mathrm{mHz}$ \\
\hline \hline
\end{tabular}

Table 1. PERFS performance indicators. 


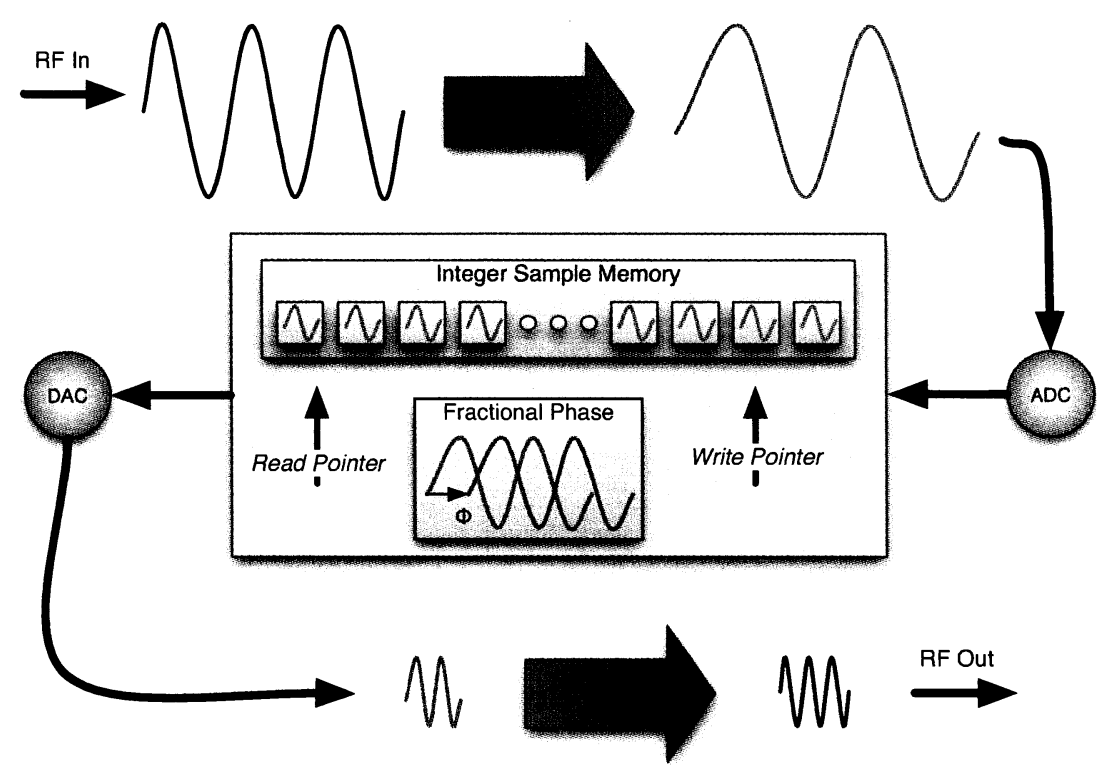

Figure 4. PERFS internal concept of operations.

$2.05 \mathrm{GHz}$ for IRAS, is down-converted to the Intermediate Frequency (IF) of $35.42 \mathrm{MHz}$ at 1 volt peak-to-peak with a $2 \mathrm{MHz}$ bandwidth. The IF is sampled by the Analog-toDigital Converter (ADC). The parameters that specify Doppler shift and delay are applied. The sample memory provides the integer delay, and the Direct Digital Synthesizer (DDS) generates the fractionally delayed and Doppler shifted signal that is converted to analog via the Digital-to-Analog Converter (DAC). Digital Step Attenuators (DSAs) provide the commanded attenuation to the IF signal. Finally, the IF signal is up-converted to RF. Additional information about specific operations, highlighted in Figure 4, follow:

IF SAMPLING: The IF input is digitized with a 12-bit ADC running at 100 MSPS, as driven by the write-pointer DDS.

Sample Memory: The ADC output is provided to a Field Programmable Gate Array (FPGA) that implements a circular memory buffer. Each integer sample in the memory buffer represents $10 \mathrm{~ns}$ of delay based on the $100 \mathrm{MHz}$ DDS clocks when no Doppler is applied.

Doppler and Delay: The Doppler shift is generated by varying the frequency of the read-pointer DDS clock output. The total instantaneous delay is a combination of the integer and fractional delay. The integer delay is provided by sample memory, while the fractional delay is represented by the phase difference between DDS clocks.

AtTenuation: Attenuation is applied via DSA in $0.5 \mathrm{~dB}$ steps, which are controlled by the FPGA. The applied attenuation includes free space loss, antenna gain and alignment, etc., as provided in the commanded parameters. The prototype PERFS is limited to a maximum range of $10 \mathrm{~km}$, while the production units will support a maximum range of approximately $3500 \mathrm{~km}$. 


\section{Testing}

To assess the IF performance of the PERFS prototype with respect to Table 1, the following tests were performed related to attenuation, Doppler, and range. They are described in more detail below.

- Attenuation

- Free space loss

- Sideband suppression

- Doppler

- Coarse range steering

- Tracking/Carrier Modulation (coarse delay)

- Resolution (fine/group delay)

- USB Interface

- Data Throughput

FREE SPACE LOSS: A sequence of ranges is commanded to the PERFS prototype and the resulting attenuation is measured with a spectrum analyzer. The measured result is then compared to the computed value for each range setting.

SIDEBAND SUPPRESSION: Signal products resulting from mixing must not interfere with the center frequency of interest. To determine the sideband suppression, an IF sine wave is input to the PERFS prototype and the output sideband peak power is measured.

COARSE RANGe STEERING: As a basic functional test, the PERFS prototype is started at its minimum range, and provided a sequence of commanded range values. The output signal is recorded and the result is post-processed to determine the range and range error.

TRACKING/CARRIER MODULATION: It is important that data modulated on the carrier frequency input are accurately reproduced on the PERFS output. Reproduction of carrier modulation and Doppler tracker is accomplished by injecting GPS Pseudo-Random Noise (PRN) $1^{\text {t† }}$ onto the IF input. The carrier frequency is then stepped by a $10 \mathrm{~Hz}$ Doppler shift every $100 \mathrm{~ms}$ for a duration of $10 \mathrm{~s}$, producing a $1 \mathrm{kHz}$ total frequency shift. The output signal is recorded and the result is post-processed to determine the range and range error.

RESOlution: It is important to know the minimum measurable change in range that the device, in sum, can support. To do this, an input IF sine wave is split. One input signal is recorded directly, without passing through the PERFS prototype. The second input signal is passed into the PERFS prototype with specified constant Doppler applied for approximately $10 \mathrm{~s}$, and the output is recorded. This is repeated for several constant Doppler values. The resulting signals are post-processed to determine phase residual and estimated measurement noise. In this post-processing, the electrical path delay is removed.

\footnotetext{
${ }^{\dagger \dagger}$ Each GPS satellite broadcasts a unique PRN code, which receivers use to identify tracked satellites. ${ }^{21}$
} 
Data Throughrut: Currently, estimates require that command and telemetry maintain a total data rate of not less than $5 \mathrm{~KB} / \mathrm{s}$, with a nominal $100 \mathrm{~ms}$ update rate. Since there are several driver options available for communicating with the FTDI chipset planned for use in PERFS, the prototype implemented the simplest of those, viz. the Virtual COM Port (VCP) drivers ${ }^{22}$ found in the default Fedora Core 5 Linux distribution via the ftdi_sio library. In

this test, an identification request is written to the device and the result is read into local buffer. This is done repeatedly over various time intervals to establish a baseline data rate.

\section{III.A. Test Equipment}

The following equipment was used in the testing:

- Network analyzer: HP 8753D

- Spectrum analyzer: HP 8561E

- Universal counter: HP 53132A

- Power meter: HP 4418B

- Time interval analyzer: Timing Solutions Corp 5110A

- Signal generators: R\&S SIMQ series (GPS), Agilent E4421B (carrier only)

- Signal recorder

- Software GPS receiver

Only the signal recorder and the software GPS receiver are non-standard test equipment. While the software GPS receiver is self-descriptive, the signal recorder requires a brief description.

The signal recorder produces an interleaved 32-bit complex sample, divided into two 16bit in-phase and quadrature words, respectively. Each signal is sampled at $2.048 \mathrm{MHz}$. Thus, any two recorded signals can be compared easily. More Detailed information about these two items can be found in Heckler and Garrison. ${ }^{23,24}$

\section{Results}

The tests described in the previous section were performed incrementally and repeated frequently during the PERFS prototype development. This approach was necessary to provide feedback and direction to transition from concept and components to an integrated prototype. The results that follow are snapshots of tests that demonstrate the viability of the design concept and efficacy of the PERFS prototype.

\section{IV.A. Attenuation}

The free space loss and sideband suppression test results are shown in Figures 5 and 6 . From Figure 5, the lower range free space loss values disagree by one DSA step. This identified an FPGA table look-up error which was later corrected. While the prototype is limited to two DSAs spanning a range of $100 \mathrm{~m}$ to $10 \mathrm{~km}$, the production unit will include an additional DSA to span the desired physical range.

Figure 6 shows that in an initial configuration, the first sideband frequency was approximately $6 \mathrm{MHz}$ and $50 \mathrm{~dB}$ below the nominal center frequency. This spur was later determined to be power supply noise and was removed, pushing the next sideband outside the $20 \mathrm{MHz}$ span to approximately $29 \mathrm{MHz}$ below peak power and significantly closer to the noise floor. 


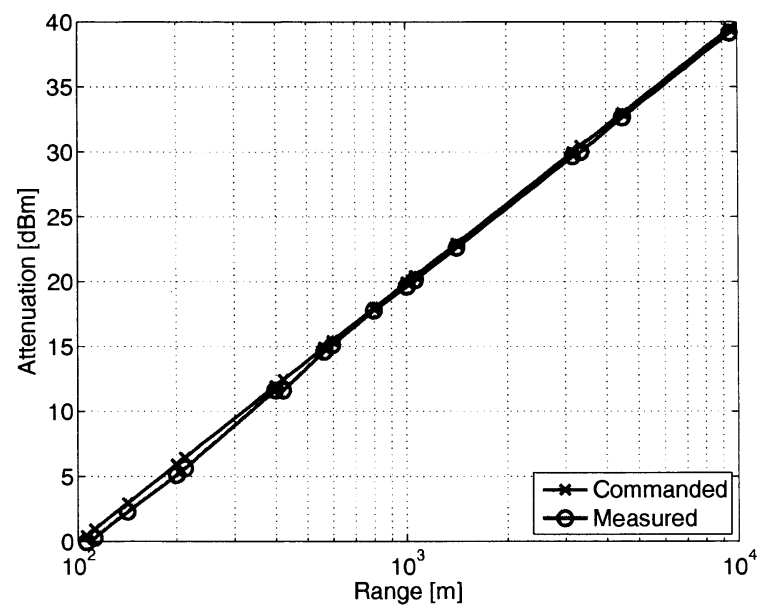

Figure 5. Commanded and measured free space loss attenuation.

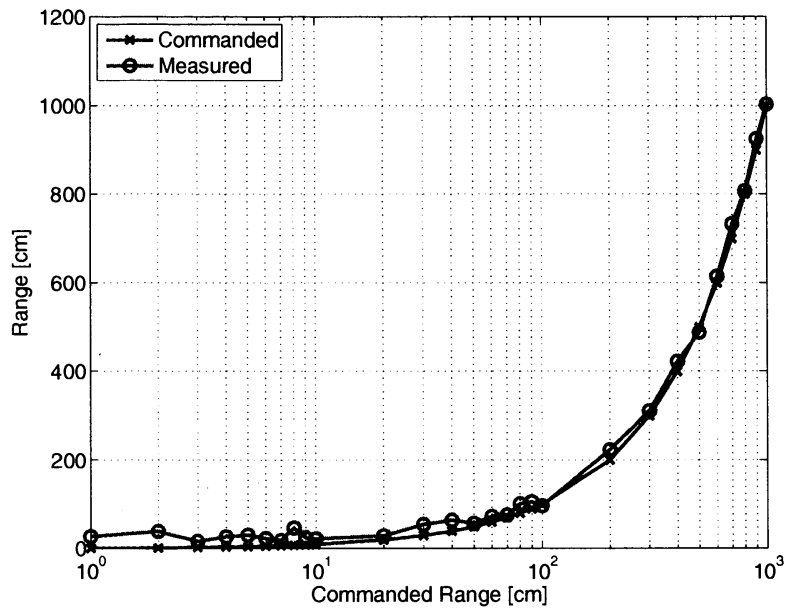

Figure 7. Doppler driven range measurement.

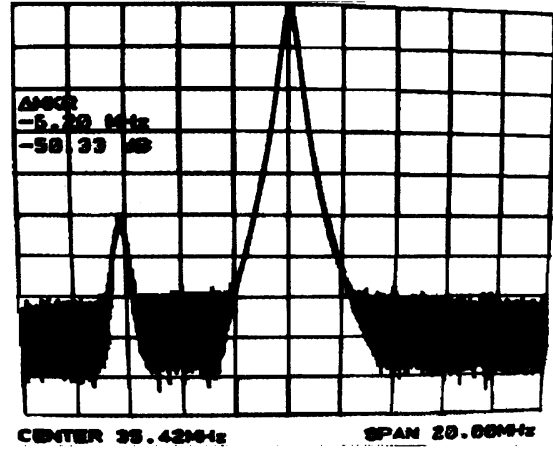

Figure 6. Sideband suppression of next strongest mixing multiple.

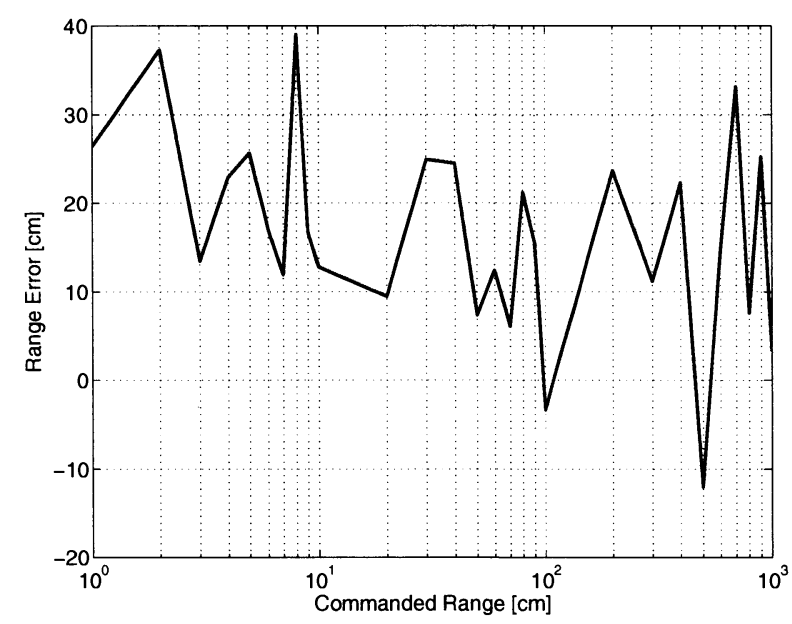

Figure 8. Doppler driven range measurement error.

\section{IV.B. Doppler}

The coarse range Doppler steering test was an early functional test. From Figures 7 and 8, the initial results clearly show that steering the range with applied Doppler was effective, and agreed to within $40 \mathrm{~cm}$ of absolute magnitude. Additionally, Figure 8 indicates that the group delay through the PERFS prototype must be determined to quantify the minimum measurable range change. This is discussed later in this section.

The tracking/carrier modulation test was used to ensure that modulated data are not corrupted by PERFS. This test required additional post-processing of the recorded signal with a software GPS receiver. The results summary for this test can be seen in Figures 9-10.

Figure 11 clearly indicates that the modulated code data (PRN 1) were successfully passed through the PERFS prototype and tracked by the software GPS receiver. The measured phase resulting from the Doppler stepping agrees well with the commanded Doppler shift. Coarse acquisition was achieved within approximately 6 steps. The code and carrier tracked range-rate can be see in Figure 12. The range rate trends agree after initial Carrier Acquisition (CA). The code tracked range shown in Figure 9 indicates a clear bias with respect to the Doppler integrated range that, again, results from initial CA. Comparing the integrated and code tracked range, Figure 10 indicates the bias is approximately $172 \mathrm{~m}$ and 


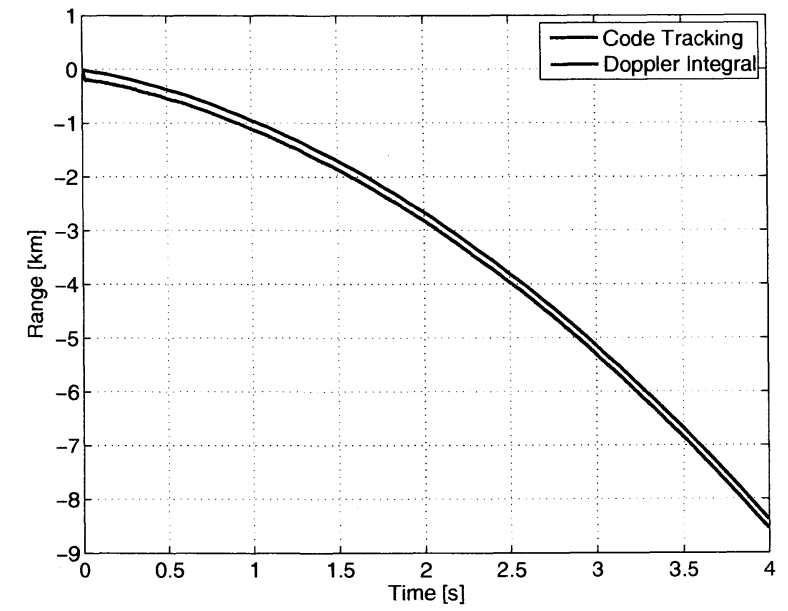

Figure 9. Code tracked and Doppler integrated range.

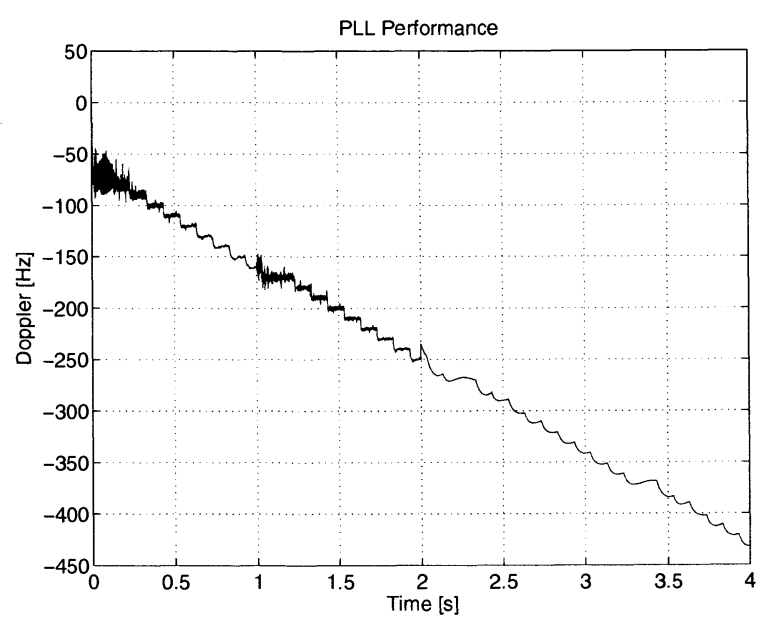

Figure 11. Phase lock loop tracking performance.

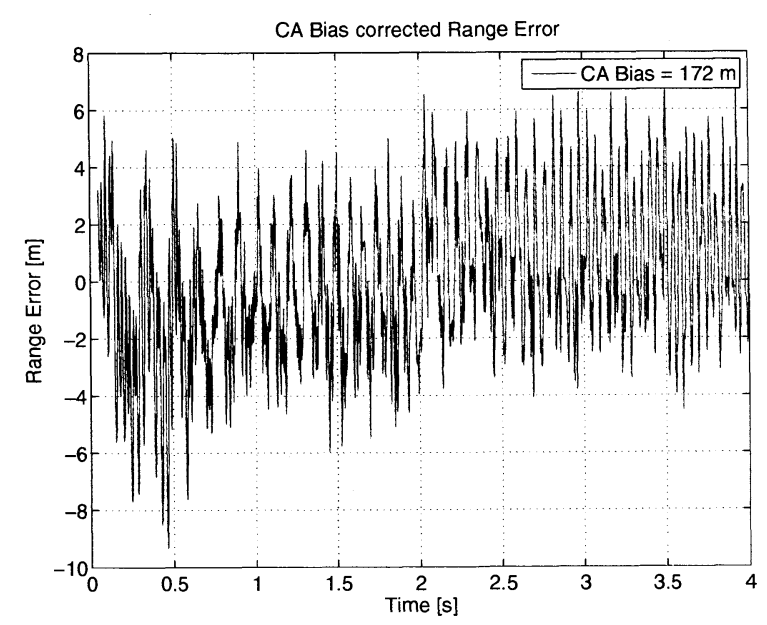

Figure 10. Biased corrected code tracked range error.

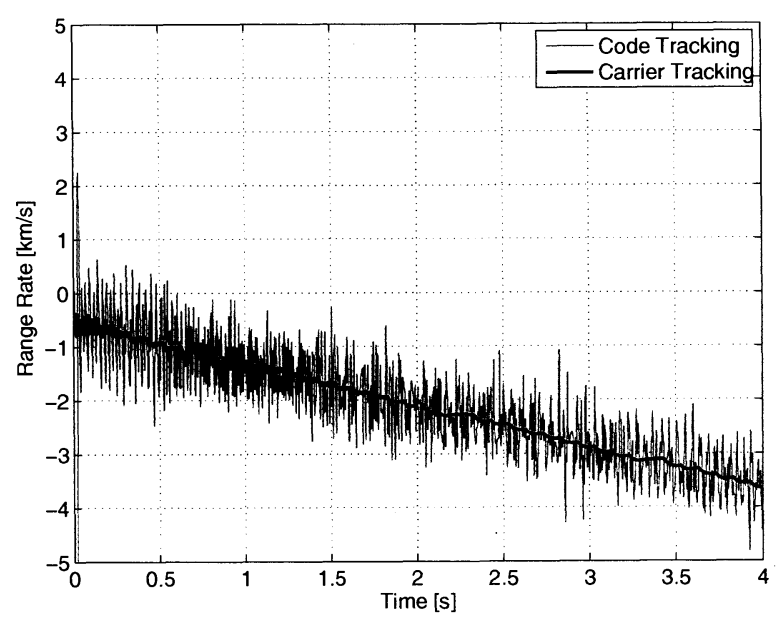

Figure 12. Code tracked and Doppler-commanded range rate.

the range error magnitude is less than $10 \mathrm{~m}$ for the duration of the test.

For group delay testing to determine the phase resolution, the phase measurement residual $\Delta \phi_{e}$ is computed as

$$
\Delta \phi_{e}=\phi_{p}-\phi_{m}
$$

where $\phi_{p}$ and $\phi_{m}$ are the linear model predicted phase and the measured phase, respectively. The double-differenced residual, $\Delta^{2} \phi_{e}$, provides an estimate of the measurement noise for the phase residual, $\Delta \phi_{e}$, and the $3 \sigma$ value of $\Delta^{2} \phi_{e}$ provides the degree of confidence. The essential results of the minimum phase resolution test are shown in Figures 13-16 and Table 2.

Figures 13 and 14 show the phase residual, as compared to a linear predictive model, for the two constant applied Doppler cases tested, $0.1 \mathrm{~Hz}$ and $1.0 \mathrm{~Hz}$, respectively. For the $10 \mathrm{~s}$ duration tested, both cases have a conservative absolute upper bound of $7^{\circ} \mathrm{IF}$. At first glance, the phase residuals do not appear related. The single-sided amplitude spectra, seen in Figure 15, show a similar frequency spacing for the lowest frequency components of each phase residual with an order of magnitude offset for the higher frequency phase residual. This is more clearly illustrated in Figure 16, in which the two phase residuals are overlaid. The phase residual for the $0.1 \mathrm{~Hz}$ applied Doppler case is shown against its full time scale of 


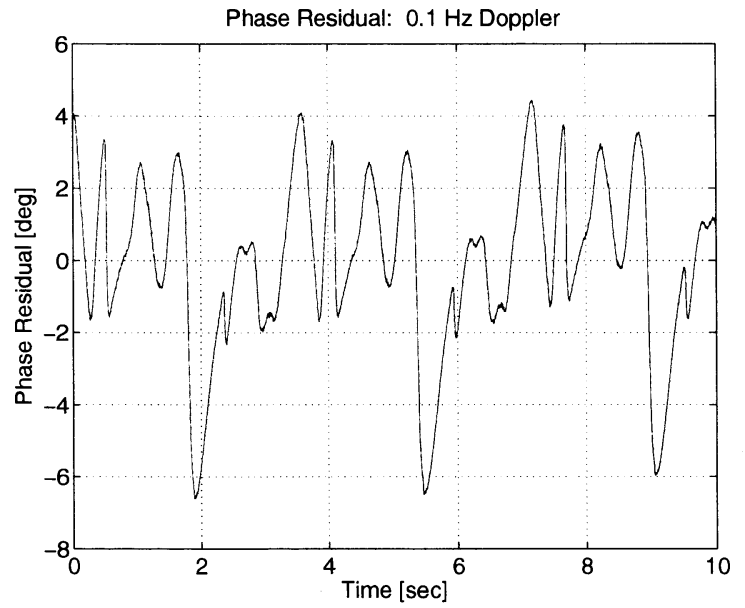

Figure 13. Phase residual for constant $0.1 \mathrm{~Hz}$ applied Doppler.

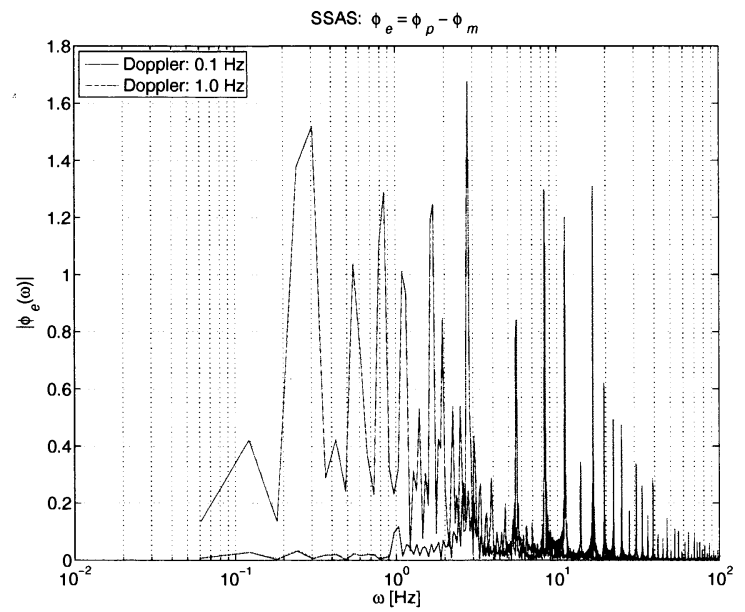

Figure 15. Phase residual single-sided amplitude spectra.

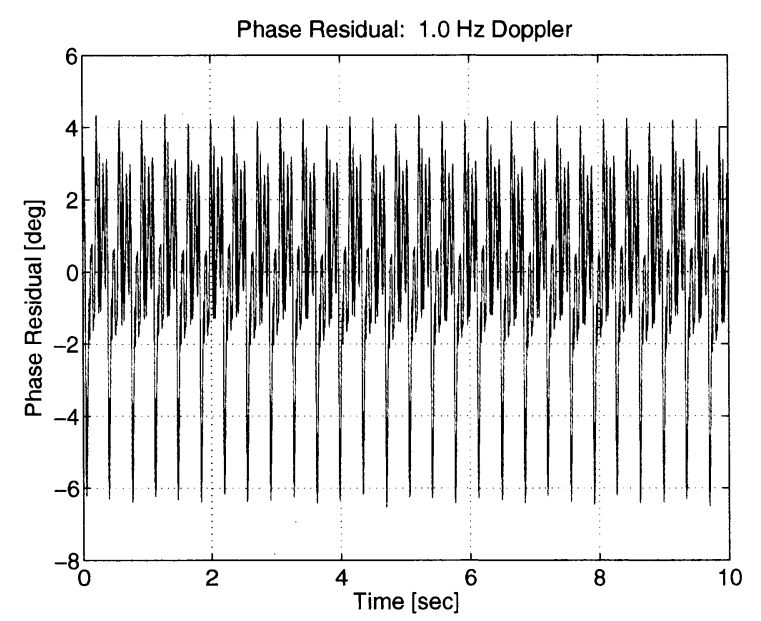

Figure 14. Phase residual for constant $1.0 \mathrm{~Hz}$ applied Doppler.

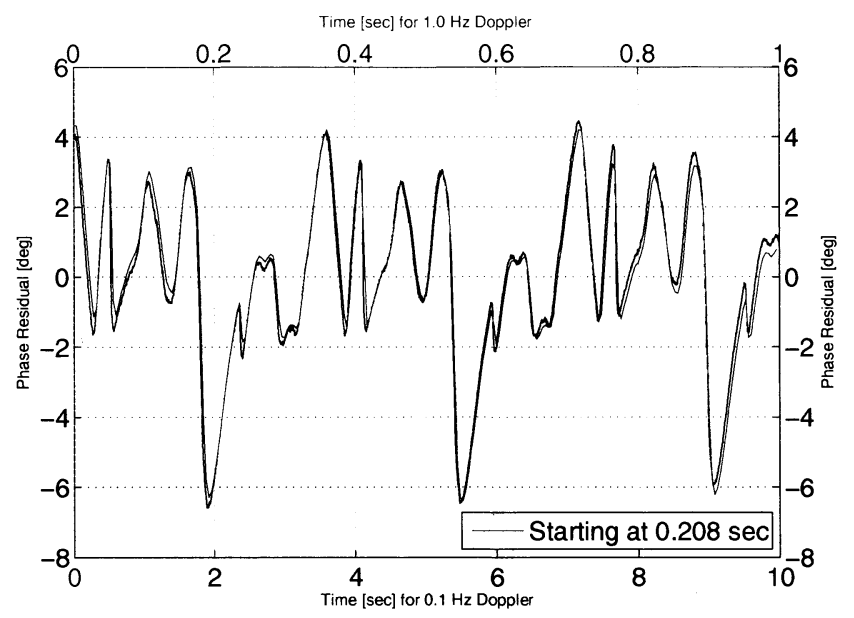

Figure 16. Offset phase residual comparison for $0.1 \mathrm{~Hz}$ and $1.0 \mathrm{~Hz}$ constant applied Doppler.

$10 \mathrm{~s}$, while the $1.0 \mathrm{~Hz}$ phase residual is overlaid for only $1 \mathrm{~s}$ with an index offset of 52 samples, which corresponds to a right shift in the data of $0.208 \mathrm{~s}$. From Figure 16, it is clear that the phase residuals possess a similar characteristic shape that scales with frequency.

Table 2 provides a summary of maximum absolute phase residual, $\max \left|\Delta \phi_{e}\right|$; estimated maximum absolute measurement noise, $\max \left|\Delta^{2} \phi_{e}\right|$; and the estimated measurement noise $3 \sigma$ confidence interval, $3 \sigma_{\Delta^{2} \phi_{e}}$. The $0.0 \mathrm{~Hz}$ Doppler case provides an indication of the noise levels and measurement resolution available. For this case, the maximum phase residual is approximately $1 \mathrm{~mm}$, with a measurement noise of the same order. From this, we conclude that the measurement resolution for the remaining tests is of the order millimeters. In the $0.1 \mathrm{~Hz}$ constant applied Doppler case, the $7^{\circ} \mathrm{IF}$ phase residual corresponds to a minimum range resolution of $16.5 \mathrm{~cm}$, and has a relatively small measurement noise. For $1 \mathrm{~Hz}$ constant applied Doppler, again, we find $16.5 \mathrm{~cm}$ as the minimum range resolution, but with significantly increased measurement noise.

Clearly, the minimum range resolution of $16.5 \mathrm{~cm}$ does not meet the requirement of $5 \mathrm{~cm}$ stated in Table 1, but the prototype performance is quite close. The common maximum phase residual and frequency dependent characteristic shape of the phase residual time history 


\begin{tabular}{ccccccc}
\hline \hline $\begin{array}{c}\text { Doppler } \\
{[\mathrm{Hz}]}\end{array}$ & \multicolumn{2}{c}{$\max \left|\Delta \phi_{e}\right|$} & \multicolumn{2}{c}{$\max \left|\Delta^{2} \phi_{e}\right|$} & \multicolumn{2}{c}{$\left.3 \sigma_{\Delta^{2} \phi_{e}} \mathrm{IF}\right]$} \\
{$[\mathrm{cm}]$} & {$\left[{ }^{\circ} \mathrm{IF}\right]$} & {$[\mathrm{cm}]$} & {$\left[{ }^{\circ} \mathrm{IF}\right]$} & {$[\mathrm{cm}]$} \\
\hline 0.0 & 0.04 & 0.1 & 0.05 & 0.12 & 0.05 & 0.12 \\
0.1 & 7 & 16.5 & 0.7 & 1.5 & 0.3 & 0.7 \\
1.0 & 7 & 16.5 & 4.0 & 10.0 & 2.0 & 5.0 \\
\hline \hline
\end{tabular}

Table 2. PERFS minimum range resolution summary.

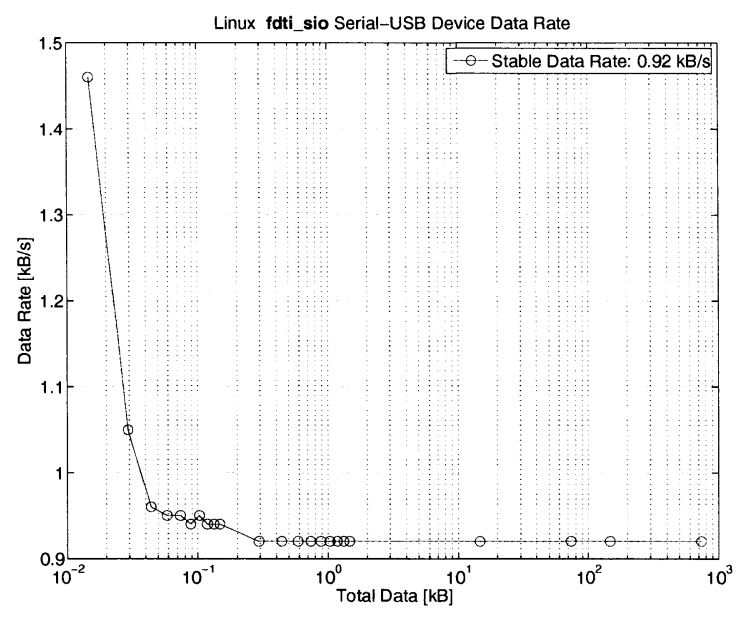

Figure 17. Maximum stabilized data-rate for FTDI FT245BL USB interface using standard Fedora Core 5 Linux ftdi_sio VCP drivers.

strongly suggest that free running clocks are the culprit, as the PERFS prototype does not have a single synchronizing clock source. It is expected that further reduction and isolation of noise sources and synchronization of internal clocks in the final design will allow the production unit to meet the desired range resolution requirement.

\section{IV.C. USB Interface}

The data rate results can be see in Figure 17. The combination of the default VCP drivers and the FTDI FT245BL chipset prototype produce a sustained data rate of less than $1 \mathrm{kB} / \mathrm{s}$. This fails to meet the production unit's expected minimum sustained data rate of $5 \mathrm{kB} / \mathrm{s}$. In the production unit, this will be remedied with a combination of a higher speed hardware interface, and if necessary, a custom device driver.

\section{Conclusions}

This work presents the concept, design, and prototype performance of a Path Emulator for Radio Frequency Signals. Characterization of the prototype device demonstrated a viable concept implementation to buffer radio frequency signals exchanged between spacecraft that accurately emulates the dynamic environment through which the the radio frequency signals travel, including the effects of medium, moving platforms, and radiated power. This capability is necessary to enable realistic hardware-in-the-loop simulations that include radio frequency ranging sensors for relative navigation. Although the prototype device itself represents only an initial design proof-of-concept, it nearly achieved many of the performance requirements intended for the final production devices. Capitalizing on information gained from this prototype testing to refine the design and function of the device, the final production devices are expected to satisfy all of the system requirements. 


\section{Acknowledgments}

The development of the Path Emulator for Radio Frequency Signals (PERFS) is supported by NASA's Office of Space Communications and Navigation (SCaN). The authors thank Gregory J. Boegner for sharing his valuable insight into the design and operation of the prototype and Gregory W. Heckler for provisioning of and assistance with the signal recorder and software GPS receiver.

\section{References}

${ }^{1}$ Aldridge, Jr. (Chairman), E. C., "A Journey to Inspire, Innovate, and Discover," Tech. rep., President's Commission on Implementation of United States Space Exploration Policy, June 2004, http://tinyurl. com/d28rx, Accessed January 23, 2007.

${ }^{2}$ Smith, D. and Colón, G., "Magnetospheric Multiscale Mission," http://stp.gsfc.nasa.gov/ missions/mms/mms.htm, Accessed January 23, 2007.

${ }^{3}$ Gendreau, K. C., Cash, W. C., Gorenstein, P., Windt, D. L., Kaaret, P., and Reynolds, C., "MAXIM: The Black Hole Imager," Proceedings of the SPIE, Vol. 5488, 2004, pp. 394-402.

${ }^{4}$ White, N. E. and Newman, P., "Micro-Arcsecond X-ray Imaging Mission," 2007, http://maxim.gsf c . nasa.gov/, Accessed January 23, 2007.

${ }^{5}$ Leisawitz, D., "Submillimeter Probe of the Evolution of Cosmic Structure," http://space.gsfc . nasa.gov/astro/specs/, Accessed January 23, 2007.

${ }^{6}$ Carpenter, K., "Stellar Imager," http://hires.gsfc.nasa.gov./ si/, Accessed January 23, 2007.

${ }^{7}$ European Space Agency, "Darwin Mission," http://tinyurl.com/86n5h, Accessed January 23, 2007.

${ }^{8}$ Swedish Space Corporation, "Prisma Mission," http://tinyurl. com/2p4z3u, Accessed June 14, 2007.

${ }^{9}$ European Space Agency, "Proba-3 Mission," http://tinyurl.com/34apzm, Accessed June 14, 2007.

${ }^{10}$ Fisher, Diane, K. and Leon, N. J., "New Millennium Program's Space Technology 9 (ST9) Project," http://nmp.jpl.nasa.gov/st9/, Accessed January 23, 2007.

${ }^{11}$ Beasley, D. and Hupp, E., "NASA Selects Advanced Technology Providers," NASA News, July 2005, http://tinyurl.com/aztru, Accessed January 23, 2007.

${ }^{12}$ Leitner, J., "A Hardware-in-the-Loop Testbed for Spacecraft Formation Flying Applications," Proceedings of the IEEE Aerospace Conference, Vol. 2, 2001, pp. 615-620.

${ }^{13}$ Naasz, B. J., Burns, R. D., Gaylor, D., and Higinbotham, J., "Hardware-in-the-Loop Testing of Continuous Control Algorithms for a Precision Formation Flying Demonstration Mission," 18th International Symposium on Space Flight Dynamics, 2004.

${ }^{14}$ Mitchell, J. W. and Luquette, R. J., "Recent Developments in Hardware-in-the-Loop Formation Navigation and Control," NASA Goddard Flight Mechanics Symposium, 2005.

${ }^{15}$ Mankins, J. C., "Technology Readiness Levels," 1995, http://www.hq.nasa.gov/office/codeq/ trl/, Accessed May 23, 2007.

${ }^{16}$ Wikipedia, "Technology Readiness Level," http://tinyurl.com/3bpug3, Accessed May 23, 2007.

${ }^{17}$ Grambling, C., "Orbit and Navigation Level 2 Requirements, Processes, and Operations Concept Document," NASA Goddard Space Flight Center, Solar Terrestrial Proves Program, MMS Mission, MMS461-OPS-0002.

${ }^{18}$ NASA GSFC Mission Engineering and Systems Analysis Division, "GEONS Open Architecture Solutions for Onboard Orbit Determination in any Orbit," 2005, http://geons.gsfc.nasa.gov/, Accessed January 23, 2007.

${ }^{19}$ Hunt, C., Smith, C., and Burns, R., "Development of a Crosslink Channel Simulator," Proceedings of the IEEE Aerospace Conference, Vol. 2, 2004, pp. 1322-1328.

${ }^{20}$ Mitchell, J. W., Zakar, D. M., Burns, R. D., and Luquette, R. J., "A Message Oriented Middleware for a Soft Real-Time Hardware-in-the-Loop Spacecraft Formation Flying Testbed," AIAA Modeling and Simulation Technologies Conference, 2006.

${ }^{21}$ National Institute of Standards and Technology, "Time and Frequency from A to Z: Global Positioning System (GPS)," http://tinyurl.com/2w4j63, Accessed May 23, 2007.

${ }^{22}$ Future Technology Devices International Ltd., "Virtual COM Port Drivers," 2007, http://www. ftdichip.com/Drivers/VCP.htm, Accessed January 23, 2007. 
${ }^{23}$ Heckler, G. W. and Garrison, J. L., "Architecture of a reconfigurable software receiver," Institute of Navigation GNSS, 2004.

${ }^{24}$ Heckler, G. W. and Garrison, J. L., "Experimental Tests of Unaided Weak Signal Acquisition Methods Using a Software Receiver," Institute of Navigation GNSS, 2006. 